\title{
Réunion de la médecine de famille et de la mé- decine d'urgence dans une vallée de montagne
}

\section{Bruno Durrera, \\ Urs Allenspach ${ }^{b}$}

a Dr, médecine générale, médecin d'urgence SSMUS, médecin du sport SSMS

b Dr, médecine générale

\footnotetext{
* Le rapport mentionné sur les modèles de soins peut être consulté sur le site Internet de la CDS www.gdk-cds.ch sous le thème Structures ambulatoires.
}

Cette série d'articles a été lancée en novembre 2012 par la Conférence des directeurs de la santé (CDS) à partir du rapport «Nouveaux modèles de soins pour la médecine de premier recours» * qu'elle a publié en février 2012 avec l'Office fédéral de la santé publique (OFSP). Les solutions décrites visent à donner des inspirations, stimuler la discussion et fournir ainsi une contribution à la promotion de la médecine de famille. Après le cabinet de groupe de médecins de campagne décentralisé et en réseau dans la région Thurtal-Untersee, le modèle de soins intégrés dans le Val Müstair et le centre médical Schlossberg, l'utilisation de synergies dans le Lauterbrunnental est décrite cidessous. nécessaire pour pouvoir continuer à assurer la couverture médicale de notre vallée de montagne à la topographie difficile. Dans notre modèle, les médecins de famille travaillent en même temps comme médecins d'urgence pour l'ambulance et l'hélicoptère médicalisé.

Une base d'hélicoptères Air-Glaciers assurant quelque 300 opérations de sauvetage par an existe depuis 1969 à Lauterbrunnen. Les patients gravement malades et blessés de Wengen, Mürren et Gimmelwald sont depuis lors évacués par hélicoptère médicalisé. Depuis 1988, les membres des associations locales de samaritains et du poste de secours du Club alpin

\section{Dans notre modèle, les médecins de famille travaillent en même temps comme médecins d'urgence pour l'ambulance et l'hélicoptère médicalisé.}

Dans le Lauterbrunnental, au cour de l'Oberland bernois, les villages de Lauterbrunnen, Stechelberg et Isenfluh sont accessibles en voiture, mais Wengen, Mürren et Gimmelwald ne le sont que par des trains de montagne. A côté d'une population permanente de quelque 3000 personnes, jusqu'à 10000 touristes sont présents durant les saisons d'hiver et d'été. De grandes manifestations sportives telles que les courses du Lauberhorn, les Infernorennen et le marathon de la Jungfrau sont des défis médico-logistiques supplémentaires. Le Jungfraujoch et le Schilthorn font également partie de la zone d'intervention. Wengen, Mürren et Lauterbrunnen ont chacun été pourvus d'un médecin depuis 1987. Depuis 2009, Mürren dispose durant la haute saison dans le centre sportif alpin d'une filiale du cabinet médical de Lauterbrunnen.

Le «Service public» en diminution et le manque croissant de médecins de famille menacent également notre région périphérique; c'est pourquoi une réunion des soins médicaux de base et d'urgence était suisse exploitent une ambulance intervenant 250 à 300 fois par an. A côté de la médecine de premier recours, les cabinets médicaux de Lauterbrunnen/Mürren et en partie de Wengen assurent en synergie également le service médical d'urgence pour l'hélicoptère médicalisé et l'ambulance. Les trois cabinets disposent de possibilités d'atterrissage par hélicoptère pour aller chercher des médecins urgentistes en service ou acheminer des patients ne nécessitant pas une hospitalisation (par ex. lors de luxations de l'épaule). Avec ce modèle, $90 \%$ de la population de la commune de Lauterbrunnen peut recevoir des premiers soins dans les 15 minutes. A l'été 2012, l'Aide Suisse aux Montagnards a de plus mis à la disposition du service d'ambulance un véhicule d'intervention du médecin d'urgence. La perspective de la combinaison stimulante de la médecine de famille et de la médecine du sport, de montagne, de sauvetage et d'urgence a jusqu'ici contribué à recruter (parfois à temps partiel) pour notre équipe des médecins d'urgence qua-

\section{Série d'articles sur les nouveaux modèles pour le cabinet de médecine de famille} dans «PrimaryCare»

Depuis le mois de février 2012, la revue PrimaryCare propose une série d'articles originaux et intéressants sur des modèles efficaces de cabinets médicaux. Vous les découvrirez sous www.primary-care.ch $\rightarrow$ Pour les lecteurs $\rightarrow$ Topic collections $\rightarrow$ Unternehmen Hausarztpraxis. 


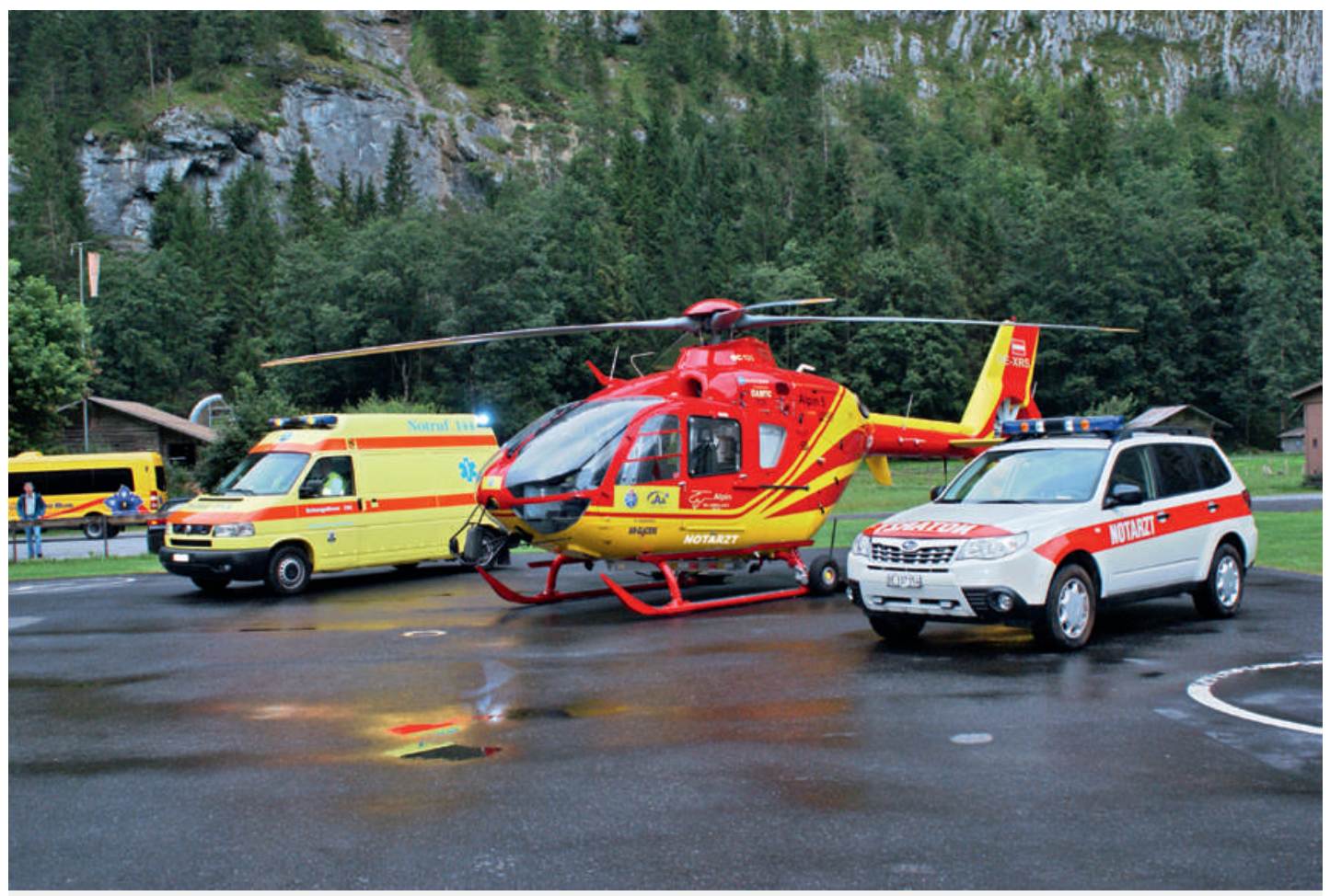

Réunion de la médecine de famille et de la médecine d'urgence dans le Lauterbrunnental: ambulance, hélicoptère médicalisé Air-Glaciers et véhicule d'intervention du médecin d'urgence.

lifiés qui aiment également faire des «consultations au bon air de la montagne» dans une superbe région de sport et d'aventure.

Le service médical d'urgence $24 \mathrm{~h}$ sur $24 \mathrm{~h}$ pour le cabinet de médecine de famille, l'hélicoptère médicalisé et l'ambulance est à ce jour organisé et financé sur une base purement privée sans aucun soutien public. La société responsable du service de médecine de famille et d'urgence Lauterbrunnen/Mürren avec les infrastructures correspondantes est depuis 1999 la Caremed SA, qui est encore entièrement propriété des médecins.

Pour l'avenir, nous sommes prudemment optimistes quant à notre modèle; nous souhaiterions toutefois à long terme une assise élargie avec davantage de soutien et de reconnaissance de la part de la politique et des financeurs. Dans les Grisons, des médecins de premier recours exerçant en périphérie sont financièrement soutenus par le canton pour assurer le service médical de garde. Pourquoi pas également dans d'autres cantons de montagne? C'est la seule façon d'encourager durablement et de développer au meilleur coût dans les vallées de montagne les synergies entre la médecine de famille et la médecine d'urgence. L'avenir des soins médicaux en périphérie se situe définitivement dans des systèmes en réseau avec des possibilités de temps partiel et qui reposent sur une assise élargie (notamment communes, réseaux de médecins, tourisme). Il ne faut pas oublier que les soins d'urgence relèvent en définitive de la responsabilité des cantons.

Aimeriez-vous présenter dans le cadre de notre série d'articles une autre initiative que vous connaissez visant à garantir et promouvoir la médecine de famille? Correspondance: Daniela Schibli, Conférence des directeurs de la santé CDS, Maison des cantons, Speichergasse 6, Code postale 684, CH-3000 Berne 7, tél. 03135620 20, fax 03135620 30, daniela.schibli[at]gdk-cds.ch 